EVIDENCE-BASED DENTISTRY

\section{The assessment of systematic reviews in dentistry}

Glenny AM, Esposito M etal. Eur J Oral Sci2003; 111: 85-92

When 65 reviews were reviewed, they had several shortcomings.

To achieve the goal of summarizing evidence of treatment effectiveness accurately, systematic reviews require rigorous methods to reduce bias and random error. This study identified 65 systematic reviews of interventions for oral, dental and craniofacial disorders, which had been published since 1990 and had reported their methods in sufficient detail.

Each review was assessed by a clinician and a methodologist. Twenty reviews focused on pain, 7 each on caries and oral medicine, 6 on periodontics and 5 each on oral surgery and TMJ disorders. The subject of 35 reviews was treatment; of 11, prevention; and of 7 , treatment with adverse events.

Assessors found that 56 reviews addressed a clearly focused question, and 48 looked for studies appropriate to their question. Quality of reviewed papers was assessed in 39 reviews, but only 25 included it in the analysis. Only 33 reviews were thought to have interpreted their results appropriately. The authors stress the importance of following established guidelines for reviews, and discuss the many difficulties of interpreting results.

\section{doi:10.1038/sj.bdj.4810332}

\section{MAXILLOFACIAL SURGERY; BEHAVIOURAL SCIENCE}

Long-term assessment of psychologic outcomes of orthognathic surgery

Lazaridou-Terzoudi T, Kiyak HA et al. J Oral Maxillofac Surg 2003; 61 : 545-552

Ten years later, patients were satisfied with the results of surgery.

In this study, 117 Danish patients who had been evaluated before and after orthognathic surgery 10-14 years earlier (OS) were re-evaluated and compared with 39 patients requesting this treatment (C1) and 92 patients needing none (C2).

In the OS group, perceived problems in oral function, teeth, appearance and interpersonal relations all reduced after surgery, and again after 10+ years. Current OS group scores were better than C1 and C2, though C1 appeared to have better scores for appearance than the initial OS scores. There were minor differences according to age, and men appeared less concerned with appearance and considered their body image better than women.

The authors conclude that orthognathic surgery improves psychosocial adjustment and quality of life, and that a patient's subjective assessment should be the basis for providing this treatment.
LOCAL ANAESTHESIA

\section{Computerized local dental anesthetic systems: patient and dentist satisfaction}

Grace EG, Barnes DM et al. J Dent 2003; 31: 9-12

A computerized system was similar to traditional local anaesthetic administration.

Computerized LA systems were developed to provide precise delivery rate and controlled volume, and while some studies have shown advantages, others have not. In this study, 26 dentists gave 157 computerized (CI) and 103 traditional injections (TI) to 260 patients receiving different treatments.

On a 1-10 scale, dentists rated mean CI anaesthesia at 9.1 for profundity and patient discomfort at 2.3; scores for TI were 9.2 and 2.3. Patients in both groups had similar previous LA experience, and after treatment gave similar mean scores for their experience of CI or TI. Patients who had received CI showed a preference for it, and some dentists considered it more effective for certain injections.

doi:10.1038/sj.bdj.4810334

SPECIAL CARE DENTISTRY; SPEECH THERAPY

A four-year longitudinal study of palatal plate therapy in children with Down syndrome: effects on oral motor function, articulation and communication preferences

\section{Carlstedt K, Henningsson G et al. Acta Odontol Scand 2003; 61: 39-46}

This treatment improved speech and other functions.

The aim of plate therapy (PT) is to alter rest position and movement of the tongue, and increase upper lip mobility and facial muscle tonus. Nine Down syndrome children who wore an acrylic plate covering the palate and incorporating stimulatory devices were compared with 11 matched Down syndrome controls (C) who did not, after a period of 4 years (at a mean age of $5.6 \mathrm{yrs}$ ). PT started in the 1st year of life for 4 , the 2 nd for 3 and the 3rd for 2 , and parents were asked to ensure children used appliances for at least $1 \mathrm{hr}$ twice daily.

The PT group showed significantly less tongue visibility, and better lip-rounding during speech and flute blowing. PT children were considered by their parents to snore less at night and open their mouths less in the day than were $C$ children by their parents. There were no differences between groups in preference for spoken language or manual sign communication.

doi:10.1038/sj.bdj.4810335 